\title{
On coupled models of anisotropic contact surfaces and their experimental validation
}

Alexander Konyukhov, Karl Schweizerhof

Universität Karlsruhe, Institut für Mechanik

Institut für Mechanik

Kaiserstr. 12, Geb. 20.30

76128 Karlsruhe

Tel.: +49 (0) 721/ 608-2071

Fax: +49 (0) 721/ 608-7990

E-Mail: ifm@uni-karlsruhe.de

www.ifm.uni-karlsruhe.de 


\title{
On coupled models of anisotropic contact surfaces and their experimental validation
}

\section{Alexander Konyukhov, Karl Schweizerhof}

2007

\begin{abstract}
.
The necessity to apply a coupled contact interface model including anisotropy for both adhesion and friction is shown via a set of experiments for a rubber surface possessing a periodical waviness, and therefore, an obvious anisotropic structure. The focus of experimental investigations is placed upon the measurements of the global macro characteristics such as global forces and trajectories of a sliding block in order to validate the proposed computational model.
\end{abstract}

\section{Keywords.}

anisotropy, adhesion, friction, contact, coupled model, experimental verification

\section{Introduction}

Smoothness and isotropy of contacting body surfaces can vary considerably for different contact problems. Classifying the surfaces roughness two types can be distinguished: a) surfaces with randomly distributed asperities, and b) asperities with algorithmic structure, e.g. the considered surface shows different macro properties in different directions.

Mechanical characteristics for the associated contact problems of the first type a) are obtained via statistically distributed asperities. Statistical analysis of a real rough surface and experimental aspects of its measurements have been developed in a series of publications: Longuet-Higgins [20], Greenwood and Williamson [9], Whitehouse and Archard [22] and more recently Whitehouse and Phillips [23] and Greenwood [10]. A comparative analysis of these surface models is presented in McCool [17]. These experimentally proved models later have been incorporated into finite element models, see e.g. Wriggers and Zavarise [25], [26], Buczkowski and Kleiber [6]. More advanced numerical analyses including homogenization methods and multi-scaled modeling are presented in Bandeira et. al. [1], [2]. Carbone and Mangialardi [7] derived contact tractions analytically for a particular example with a rigid wavy surface with a sinusoidal profile, assuming the presence of an adhesion hysteresis for $2 \mathrm{D}$ plane strain elasticity problem.

Constitutive modeling is applied for problems of the second type b). Such models are based on the generalization of Coulomb's friction law into the 
anisotropic domain. One of the first models has been proposed by Michalowski and Mroz [18] considered the sliding of a rigid block on an inclined surface. A model of orthotropic friction has been analyzed and consistently developed in Zmitrowicz [28], Curnier [8]. Various cases of anisotropy were presented in He and Curnier [11] based on the theory of tensor function representations and in Zmitrowicz [29] based on consideration of a relative sliding velocity. In the latter contribution, a classification of anisotropic surfaces based on the number of eigenvalues of the friction tensor has been proposed.

When looking at practical problems concerning contact interactions with friction between bodies made of soft rubber-like materials there are some situations in which the tangential elasticity of the contact surfaces should be taken into account. In these cases anisotropy for elastic forces (adhesion) and frictional forces might be coupled. Such a model including coupling of anisotropy for both friction and adhesion has been developed and analyzed numerically in Konyukhov and Schweizerhof [14], [15]. In the current contribution we discuss the validation of this model with a particular experimental test. The contact surfaces are chosen to possess visual orthotropic properties, thus a corrugated rubber mat is taken. The results of the experiments show the necessity to use the coupled model including anisotropy for both friction and adhesion. Some originally surprising experimental phenomena, such as geometrically isotropic observed behavior of a sliding block despite obvious physical anisotropies can be explained only within the proposed model.

\section{Experimental investigation}

A series of experimental tests are performed in order to investigate the global characteristics of the system "block on a rough surface". The rough surface possesses visually a clear periodical structure and, therefore, the mechanical constitutive model for an observable orthotropic structure can be applied. Since we are trying to verify the average interface model, the measured values in experiments are intentionally chosen to be global, namely we measure global forces leading to the macro friction coefficients and trajectory of a block instead of micro friction coefficients and corresponding stiffnesses of asperities. The focus of the discussion is placed upon the kinematical behavior of the block driven by a constant force together with the measurement of force components leading to this motion. Therefore, the main measurable characteristics during these experiments are global forces and trajectories of the block, which create a main basis for further calibration of models for orthotropic friction. For the judgment of the results Coulomb like models are assumed a-priori to be 
valid for the global behavior, i.e. that the tangential driving global force $F$ is proportional to the normal reaction $N: F=f(x, y) N$, where a function $f(x, y)$ describes the orthotropic properties of a surface.

\subsection{Experimental setup}

A massive block positioned on a plane is moved with constant velocity by a sliding carriage guided by rods on both sides, see Fig. 1. The block made from steel has dimensions $110 \times 110 \mathrm{~mm}$ in plane and $20 \mathrm{~mm}$ height. The mass is $m=1.875 \mathrm{~kg}$. The contact surface between the sliding carriage and the block is covered with a Teflon ${ }^{(R)}$ strip to minimize the friction between them due to relative sliding. In contrast to this, the contact surface of the steel block is covered by a suede-like material with square $90 \times 90 \mathrm{~mm}$ to increase the interaction between the block and the basement. A constant driving velocity is achieved by a step motor acting on a rack, which allows a straight displacement of $500 \mathrm{~mm}$. The contact force between the rack and the sliding carriage is measured by a force sensor. The displacement of the block during sliding is captured by an optoelectronic device which is installed on a tripod above the surface. The corresponding LED (light-emitting diode) is fixed on the block.

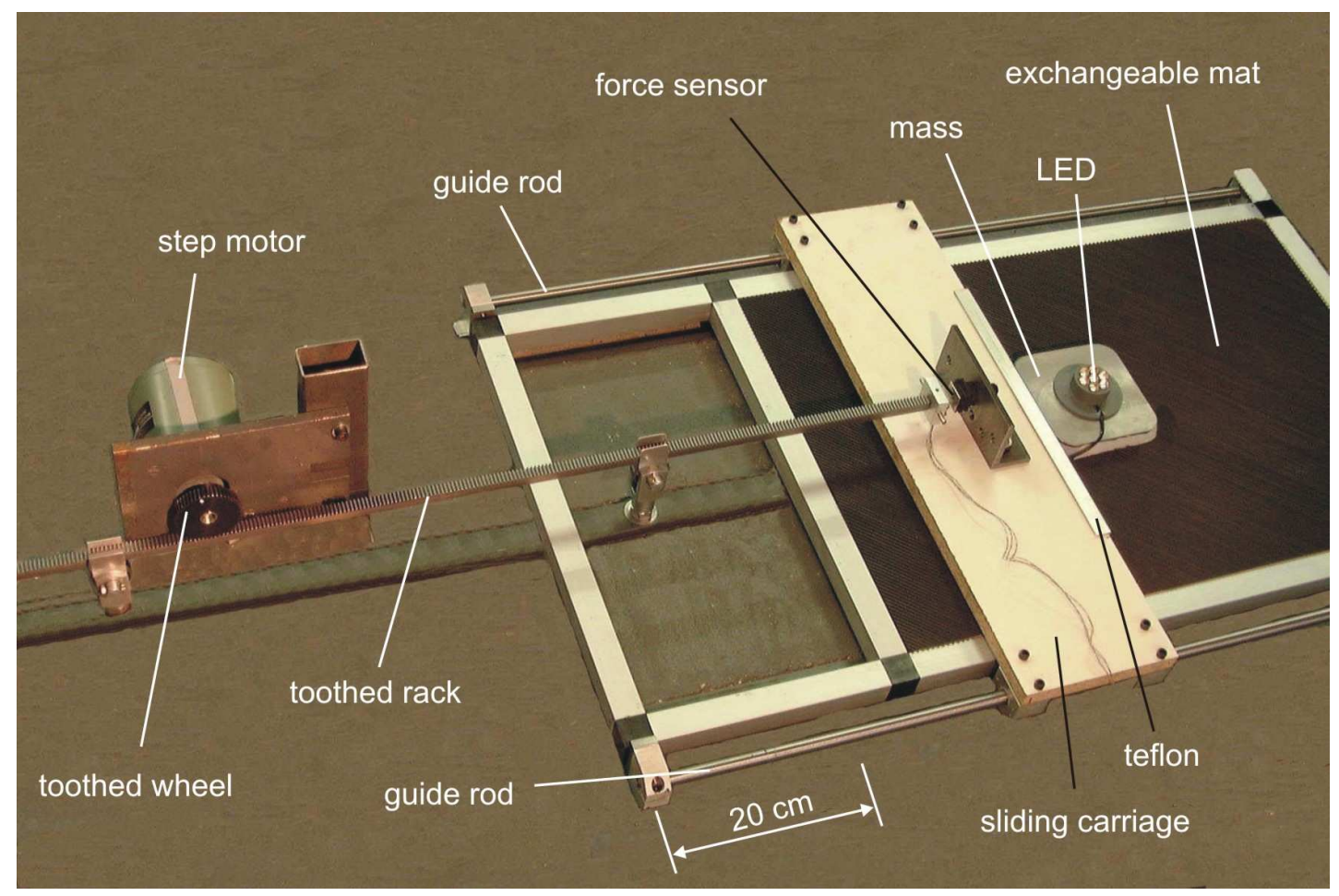

Figure 1: View on experimental setup.

For the first set of experiments a rubber mat with rather stiff ripples has been taken. Naturally it was represented by an aged corrugated rubber map. The 
frictional orthotropy is given by the wavy profile of the contact rubber surface with parallel ripples possessing in the cross section a periodical structure, see the CAD model in Fig. 2. The distance between the ripples is rather small in comparison to the dimension of the contact area of the block allowing approximately 30 ripples in contact area depending on orthotropy angle in experiments. The orientation of the ripples with respect to the fixed driving direction can be varied from $0^{\circ}$ up to $90^{\circ}$ by repositioning the mat, see Fig. 3. Again we should mention that the aim of the experiments is to show the necessity of a coupled anisotropic model for adhesion and friction, therefore, we intentionally skip any measurement of ripple stiffnesses concentrating on finding the global macro characteristics of the coupled behavior.

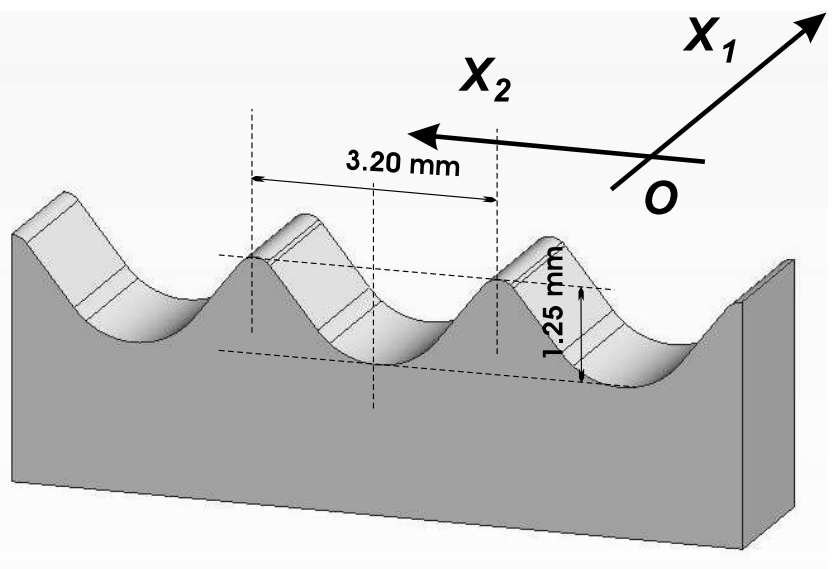

Figure 2: Geometrical structure of the corrugated rubber mat.

Wavy periodical profile, CAD model.

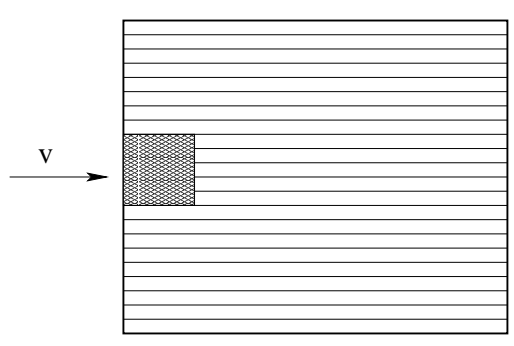

(a)

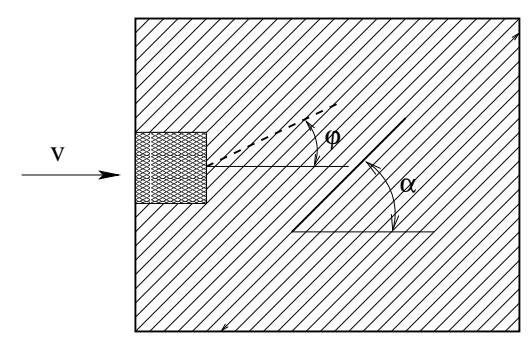

(b)

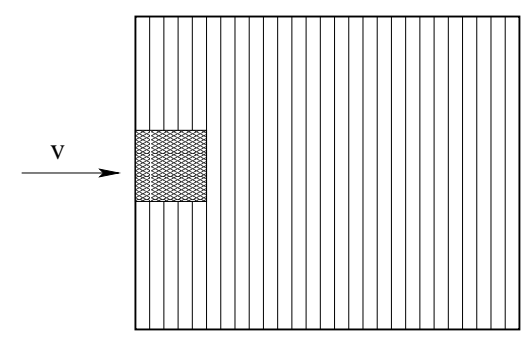

(c)

Figure 3: Orientation of the orthotropy with respect to the fixed direction of the velocity: a) $\alpha=0^{\circ}$, b) $\alpha=0^{\circ}<\alpha<90^{\circ}$, c) $\alpha=90^{\circ}$. The trajectory of the block is a straight line declined at angle $\varphi$. 


\subsection{Experimental results}

At the beginning some experiments are performed to find out the global macro mechanical properties of the system. All experiments were reproduced with a driving velocity of the carriage $v=24.4 \mathrm{~cm} / \mathrm{sec}$. First of all, the sliding carriage was moved without the block in order to define the internal resistant force $F_{\text {int }}$. Then experiments have been made with the sliding block to define the resulting driving force in the case $\alpha=0^{\circ}$, corresponding to the $X_{1}$-axis along the ripples, see CAD model in Fig. 2, and $\alpha=90^{\circ}$, corresponding to the $X_{2}$-axis across the ripples, see CAD model in Fig. 2, respectively. These measurements together with subtracting the internal force lead to the definition of macro friction coefficients $\mu_{1}$ and $\mu_{2}$ corresponding to angles $\alpha=0^{\circ}$ and $\alpha=90^{\circ}$. Assuming Coulomb friction law the friction coefficients $\mu_{i}$ were computed as

$$
\begin{aligned}
& \mu_{1}=\frac{F_{\alpha=0^{\circ}}-F_{\text {int }}}{N}=\frac{12.50-5.00}{1.875 \cdot 9.806}=0.408 \approx 0.41 \\
& \mu_{2}=\frac{F_{\alpha=90^{\circ}}-F_{\text {int }}}{N}=\frac{16.50-5.00}{1.875 \cdot 9.806}=0.625 \approx 0.63 .
\end{aligned}
$$

We note for further references that the macro friction coefficient across the ripples is found to be higher than the macro friction coefficient along ripples.

The second set of experiments to find macro parameters of the interface model is made by setting the angle $\alpha$ varying in steps from $\alpha=0^{\circ}$ up to $\alpha=90^{\circ}$. The focus lies on the definition of the trajectory of the sliding block. In all cases, the trajectory was observed as a straight line inclined with the angle $\varphi=\varphi(\alpha)$, see sketch in Fig. 3(b). The mean value $\varphi$ after 10 experiments for each angle $\alpha$ is taken for the representation. Combining all results leads to the diagram in Fig. 4 showing the dependency of the inclination angle $\varphi$ on the orientation of the orthotropy given by the angle $\alpha$ of the ripples. The maximum of the inclination angle $\varphi$ is located in the range of small angles $\alpha$ (the maximum is on the left side on the graph).

\subsubsection{Geometrically isotropic observed behavior of a sliding block}

As a fairly surprising result detected in the experiments a large sensitivity to the elastic properties of the rubber ripples was obtained. Thus, if the rubber mat with highly elastic rubber ripples (e.g. a new mat) has been taken for the second set of experiments, then the inclination angle $\varphi$ was only varying in a very small range about $0 \leq 2^{\circ}$. However, the measurement of forces still showed the difference between the macro coefficients of friction $\mu_{1}$ and $\mu_{2}$. We observe that macro friction orthotropy is still present, but the kinematical effect of the orthotropy disappears. 


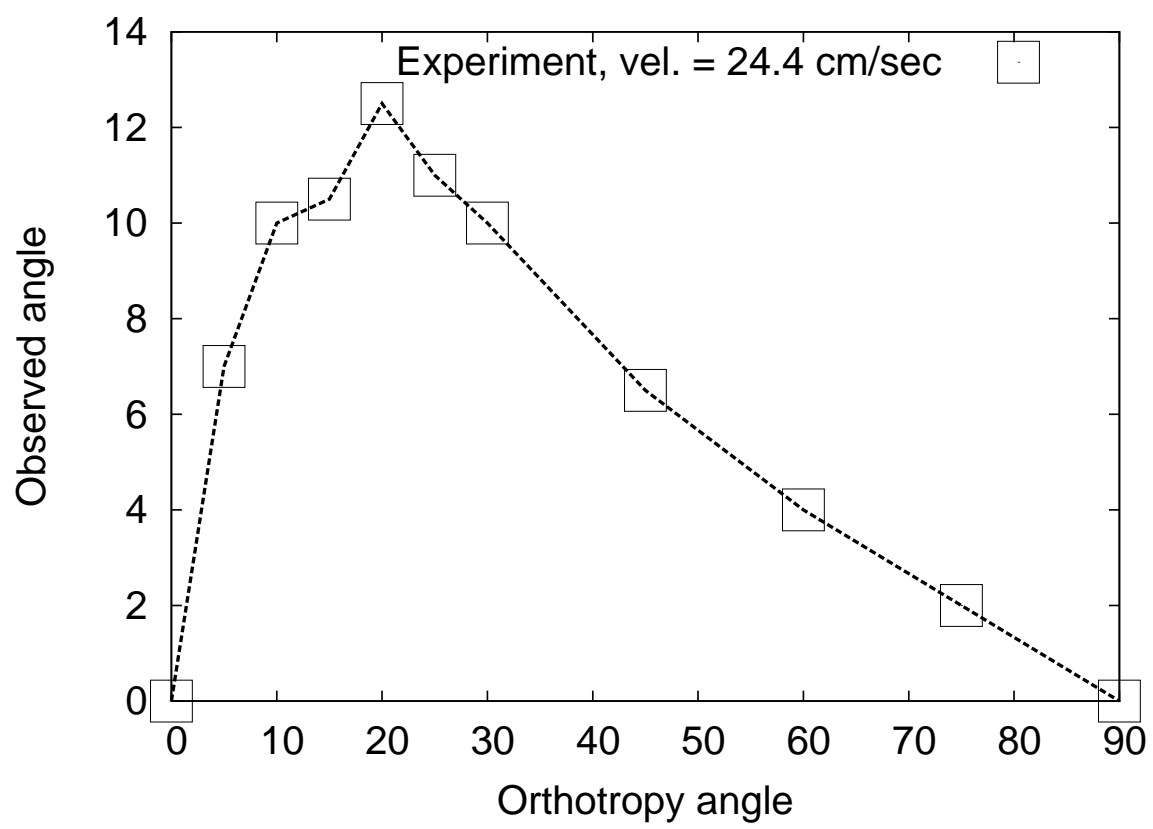

Figure 4: Observed mean value of the inclination angle $\varphi$ vs. orthotropy angle $\alpha$. Experimental results for different velocities of the block.

As we show later, the orthotropic friction model is not capable to describe this effect from the macro-model point of view, but the coupled orthotropic adhesion - orthotropic friction interface model allows to qualitatively describe the observed phenomena.

\section{Analysis of various models for anisotropic friction and applicabil- ity to the observed phenomenon.}

In this section, the range of applicability of a classical model of orthotropic friction, based only on the orthotropic friction tensor and its generalization including orthotropy for both friction (inelastic region) and adhesion (elastic region) is discussed. The necessity to assume in addition elastic properties for the surface will be shown.

As a first simple model which can be investigated analytically a material point on a plane is considered. According to the experimental tests we assume a quasi-statical motion of the material point $\mathbf{A}$ with weight $P$ loaded by the force $\mathbf{F}$ acting along the $X^{1}$-axis, see Fig. 5. The orthotropic properties of the surface are defined in the coordinate system $\xi^{1}, \xi^{2}$ inclined with an angle $\alpha$ to the original coordinate system. During quasi-statical loading, point $\mathbf{A}$ is moving along a line with velocity vector $\mathbf{v}$ inclined with an angle $\varphi$. The reaction force $\mathbf{T}$ with Cartesian components $T_{1}, T_{2}$ is acting on the point. The values of components depend on the hypothesis concerning the orthotropic friction law. Here 
two variants of the orthotropic law are considered: the well known orthotropic friction Coulomb law and a contact interface model including orthotropy for both friction and adhesion, see Konyukhov and Schweizerhof [14], [15].

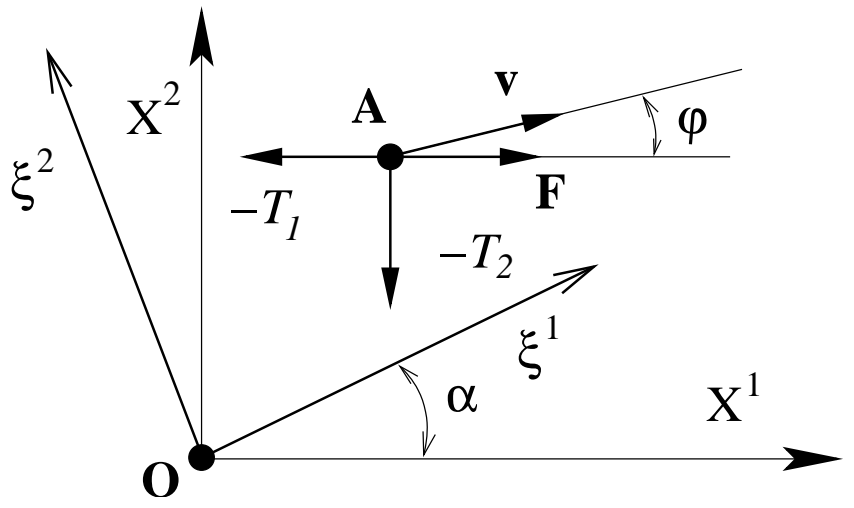

Figure 5: Motion of material point $\mathbf{A}$ on an orthotropic plane loaded by force $\mathbf{F}$.

The equilibrium equations for the system in Fig. 5 are given as:

$$
\left\{\begin{array}{l}
X^{1}: F+T_{1}=0 ; \\
X^{2}: T_{2}=0 \\
X^{3}:-P+N=0 .
\end{array}\right.
$$

where $N$ is the reaction force along the $X^{3}$ axis.

The principle of maximum dissipation is applied to obtain relation between the sliding force $\mathbf{T}$ and sliding displacements $\Delta \mathbf{r}^{s l}$. This principle requires that the dissipation function $\Psi$ reaches its maximum

$$
\Psi:=\Delta \mathbf{r}^{s l} \cdot \mathbf{T}=\Delta x_{s l}^{i} T_{i} \longrightarrow \max ,
$$

where $\Delta \mathbf{r}^{s l}$ is an increment of the sliding vector. The dissipation function $\Psi$ must also satisfy the sliding condition, formulated via inequalities, reflecting the assumed friction law, e.g. Coulomb's law.

\subsection{Orthotropic Coulomb friction law.}

First, we recall the standard case known in literature, see e.g. [18], [8], [19], [29], where orthotropy is defined only for the sliding forces. The model is formulated according to the generalization of the sliding criteria. The yield function for the Coulomb friction law is then written as

$$
\Phi:=\sqrt{\mathbf{T} \cdot \mathbf{F T}}-|N|=\sqrt{T_{i} T_{j} f^{i j}}-|N| .
$$

The sticking and the sliding conditions are defined by the rule:

$$
\Phi<0 \rightarrow \text { sticking; } \Phi \geq 0 \rightarrow \text { sliding } .
$$


According to equations (4-5) the material point is not moving during sticking (no adhesion) and the motion starts when $\Phi=0$. The components of the friction tensor $f^{i j}$ are defined for the orthotropy on the plane via e.g. the spectral representation plane as follows:

$$
\begin{aligned}
\mathbf{F} & =\mathbf{Q}_{\alpha} \boldsymbol{\Lambda}_{F} \mathbf{Q}_{\alpha}^{T}= \\
& =\left[\begin{array}{cc}
\cos \alpha & -\sin \alpha \\
\sin \alpha & \cos \alpha
\end{array}\right] \cdot\left[\begin{array}{cc}
\frac{1}{\mu_{1}^{2}} & 0 \\
0 & \frac{1}{\mu_{2}^{2}}
\end{array}\right] \cdot\left[\begin{array}{cc}
\cos \alpha & -\sin \alpha \\
\sin \alpha & \cos \alpha
\end{array}\right]^{T} \\
& =\left[\begin{array}{cc}
\frac{1}{\mu_{1}^{2}} \cos ^{2} \alpha+\frac{1}{\mu_{2}^{2}} \sin ^{2} \alpha & \left(\frac{1}{\mu_{1}^{2}}-\frac{1}{\mu_{2}^{2}}\right) \sin \alpha \cos \alpha \\
\left(\frac{1}{\mu_{1}^{2}}-\frac{1}{\mu_{2}^{2}}\right) \sin \alpha \cos \alpha & \frac{1}{\mu_{1}^{2}} \sin ^{2} \alpha+\frac{1}{\mu_{2}^{2}} \cos ^{2} \alpha
\end{array}\right],
\end{aligned}
$$

where $\mu_{i}>0$ are friction coefficients along the axis $\xi^{i}$ inclined at angle $\alpha$.

The standard method of the convex analysis is applied to obtain the sliding forces with regard to the principle of maximum dissipation (3). Thus, the Lagrange function with the multiplier $\lambda$ is specified as

$$
\mathcal{L}:=-\Psi+\lambda \Phi=-\Delta x_{s l}^{i} T_{i}+\lambda\left(\sqrt{T_{i} T_{j} f^{i j}}-|N|\right)
$$

together with the complementary Kuhn-Tucker conditions:

$$
\lambda \geq 0, \quad \lambda \Phi=0 .
$$

The optimality conditions $\frac{\partial \mathcal{L}}{\partial T^{i}}=0$ lead to the following sliding displacement components:

$$
\Delta x_{s l}^{i}=\lambda \frac{T_{j} f^{i j}}{\sqrt{T_{k} T_{l} f^{k l}}} .
$$

These equations recover the trajectory of a block as a straight line declined by angle $\varphi$, which is confirmed by experiments.

Now, taking into account the second equilibrium equation (2) $\tan \varphi$ can be determined:

$$
\left.\begin{array}{l}
\Delta x^{1}=\lambda \frac{T_{1} f^{11}}{\sqrt{T_{k} T_{l} f^{k l}}}, \\
\Delta x^{2}=\lambda \frac{T_{1} f^{12}}{\sqrt{T_{k} T_{l} f^{k l}}},
\end{array}\right\} \Longrightarrow \tan \varphi=\frac{\Delta x^{2}}{\Delta x^{1}}=\frac{f^{12}}{f^{11}}
$$


and, after transformations taking into account the values determined in eqn. (6), we finally obtain:

$$
\tan \varphi=\frac{\left(\mu_{2}^{2}-\mu_{1}^{2}\right)}{\mu_{2}^{2}+\mu_{1}^{2} \tan ^{2} \alpha} \tan \alpha
$$

\subsection{Model for orthotropic contact interfaces including both adhesion and friction.}

An alternative model involving coupling orthotropy for both adhesion and friction can be proposed including the elastic-plastic analogy and the returnmapping scheme. This model is investigated theoretically and developed into the computational model by Konyukhov and Schweizerhof [14], [15]. Then the problem is formulated in continuous form as follows

a) The relative velocity vector of the contact point is decomposed additively into an elastic part $\mathbf{v}^{e l}$ and a sliding part $\mathbf{v}^{s l}$

$$
\mathbf{v}^{r}=\mathbf{v}^{e l}+\mathbf{v}^{s l} .
$$

b) The elastic part $\mathbf{v}^{e l}$ is responsible for reversible deformations (adhesion) and satisfies the evolution equations

$$
\frac{d \mathbf{T}}{d t}=\mathbf{B v}^{e l} .
$$

At this point an adhesion tensor $\mathbf{B}$ describing orthotropic properties for the elastic region is introduced.

c) The tangential force $\mathbf{T}$ must satisfy the following inequalities defined via the yield function, which in tensor form can be written as:

$$
\Phi:=\sqrt{f^{i j} T_{i} T_{j}}-|N|=\sqrt{\mathbf{T} \cdot \mathbf{F T}}-|N|:
$$

- if $\Phi<0$ then the contact point is inside the elastic domain and $\mathbf{T}=\mathbf{T}^{e l}$ is an elastic force,

- if $\Phi=0$ then the contact point is sliding and $\mathbf{T}=\mathbf{T}^{s l}$ is a sliding force.

d) The power of the sliding forces, described by the energy dissipation function $D$ achieves its maximum:

$$
D:=\dot{x}_{s l}^{i} T_{i}^{s l}=\mathbf{v}^{s l} \cdot \mathbf{T}^{s l}, \quad D \longrightarrow \max .
$$


The principle of maximum dissipation requires that the plastic dissipation function $D$ subjected to the inequality conditions (14) achieves a maximum. For the computational treatment, the model is reformulated in incremental form and then the return-mapping scheme is applied. The incremental analog is given as

i) The full incremental displacement vector $\Delta x^{i}=\Delta x_{(n+1)}^{i}-\Delta x_{(n)}^{i}$ is decomposed additively into an elastic increment $\Delta x_{e l}^{i}$ and into a sliding increment $\Delta x_{s l}^{i}$ :

$$
\Delta x^{i}=\Delta x_{e l}^{i}+\Delta x_{s l}^{i} .
$$

ii) The elastic increment $\Delta x_{e l}^{i}$ is computed via the incremental evolution equations, for which the tensor $\mathbf{B}$ is assumed to be constant:

$$
T_{i(n+1)}^{t r}=b_{i j} \Delta x_{e l}^{i(n+1)}=b_{i j}\left(x_{e l}^{i(n+1)}-x^{i(0)}\right) .
$$

iii) In order to decide whether the trial force $\mathbf{T}^{t r}$ is a sliding force $\mathbf{T}^{s l}$ or a sticking force $\mathbf{T}^{s t}$ the yield condition is checked in each load step:

$$
\Phi^{t r}:=\sqrt{f^{i j} T_{i(n+1)}^{t r} T_{j(n+1)}^{t r}}-N_{(n+1)}
$$

- If $\Phi^{t r}<0$ then the trial force is a real sticking force $\mathbf{T}=\mathbf{T}^{t r}$.

- If $\Phi^{t r} \geq 0$ then the sliding force must be obtained via the maximum of the energy dissipation function given in incremental form.

iv) The incremental analog of the continuous formulation eqn. (15) is then:

$$
D_{\min }^{(n+1)}:=-\Delta \mathbf{r}^{s l} \cdot \mathbf{T}_{(n+1)}^{s l}=-\Delta x_{s l}^{i} T_{i(n+1)}^{s l}, \quad D_{\min }^{(n+1)} \longrightarrow \min .
$$

We recall the results obtained in [14], [15]. There the sliding force $\mathbf{T}^{s l}$ can be defined after the necessary transformations as

$$
\mathbf{T}^{s l}=-\frac{\mathbf{B F T}^{t r}}{\sqrt{\mathbf{B F T}^{t r} \cdot \mathbf{F B F T}}{ }^{t r}}|N| .
$$

Now, we must follow the return-mapping scheme in order to define the inclination angle $\varphi$. The problem is considered as a displacement driven one, therefore the incremental displacement $\Delta \mathbf{r}=\left\{\Delta x^{1}, \Delta x^{2}\right\}$ is applied. Thus, in each load step the sliding force in eqn. (20) is computed as:

$$
\mathbf{T}^{s l}=-\frac{\mathbf{B F B} \Delta \mathbf{r}}{\sqrt{\mathbf{B F T}^{t r} \cdot \mathbf{F B F T}}{ }^{t r}}|N|=\mathbf{A} \Delta \mathbf{r} .
$$


Now, if sliding is assumed, the second component of the sliding force $T_{2}$ in the formulation depicted in Fig. 5 becomes zero, see equilibrium eqn. (2). Thus, the displacement vector components $\Delta x^{1}, \Delta x^{2}$ are coupled via the equation:

$$
T_{2}=0 \Longrightarrow a_{21} \Delta x^{1}+a_{22} \Delta x^{2}=0,
$$

leading to the equation for the angle $\varphi$ :

$$
\tan \varphi=\frac{\Delta x^{2}}{\Delta x^{1}}=-\frac{a_{21}}{a_{22}}
$$

\subsubsection{Analysis of the model by general spectral representation.}

In order to calibrate later a theoretical curve $\varphi(\alpha)$ from the experimental tests presented in Fig. 4, we consider a spectral decomposition of the matrix $\mathbf{A}$ given in eqn. (21) as

$$
\mathbf{A}=\left[a_{i j}\right]=\left[\begin{array}{cc}
\lambda_{1}^{2} \cos ^{2} \alpha+\lambda_{2}^{2} \sin ^{2} \alpha & \left(\lambda_{1}^{2}-\lambda_{2}^{2}\right) \sin \alpha \cos \alpha \\
\left(\lambda_{1}^{2}-\lambda_{2}^{2}\right) \sin \alpha \cos \alpha & \lambda_{1}^{2} \sin ^{2} \alpha+\lambda_{2}^{2} \cos ^{2} \alpha
\end{array}\right],
$$

leading together with the condition (23) to the observed sliding angle $\varphi$ defined as

$$
\tan \varphi=-\frac{a_{21}}{a_{22}}=-\frac{\left(\lambda_{1}^{2}-\lambda_{2}^{2}\right) \sin \alpha \cos \alpha}{\lambda_{1}^{2} \sin ^{2} \alpha+\lambda_{2}^{2} \cos ^{2} \alpha}=-\frac{\left(\lambda_{1}^{2}-\lambda_{2}^{2}\right) \tan \alpha}{\lambda_{1}^{2} \tan ^{2} \alpha+\lambda_{2}^{2}} .
$$

An analysis for extremal values gives us

$$
\frac{d \tan \varphi}{d \tan \alpha}=0, \Longrightarrow\left(\lambda_{1}^{2}-\lambda_{2}^{2}\right)\left(\lambda_{2}^{2}-\lambda_{1}^{2} \tan ^{2} \alpha\right)=0 .
$$

The first bracket leads to the isotropic case, whereas from the second one the following critical value is obtained:

$$
\tan \alpha_{e x t}=\frac{\lambda_{2}}{\lambda_{1}}
$$

leading to the extremum of the observed inclination angle $\varphi_{\text {ext }}$ for the motion of the point

$$
\tan \varphi_{e x t}=\frac{\lambda_{2}^{2}-\lambda_{1}^{2}}{2 \lambda_{1} \lambda_{2}}
$$

Considering the last equation (28) we can obtain a critical ratio of the eigenvalues

$$
\text { ratio }_{\text {ext }}=\frac{\lambda_{1}}{\lambda_{2}}=-\tan \varphi_{\text {ext }} \pm \sqrt{\tan \varphi_{\text {ext }}^{2}+1} .
$$

This value will be used during the validation procedure. 
For further considerations we adopt the spectral decomposition also for the adhesion tensor

$$
\begin{aligned}
\mathbf{B} & =\mathbf{Q}_{\alpha} \boldsymbol{\Lambda}_{B} \mathbf{Q}_{\alpha}^{T}= \\
& =\left[\begin{array}{cc}
\cos \alpha & -\sin \alpha \\
\sin \alpha & \cos \alpha
\end{array}\right] \cdot\left[\begin{array}{cc}
-\varepsilon_{1} & 0 \\
0 & -\varepsilon_{2}
\end{array}\right] \cdot\left[\begin{array}{cc}
\cos \alpha & -\sin \alpha \\
\sin \alpha & \cos \alpha
\end{array}\right]^{T}= \\
& =\left[\begin{array}{ll}
\varepsilon_{1} \cos ^{2} \alpha+\varepsilon_{2} \sin ^{2} \alpha & \left(\varepsilon_{1}-\varepsilon_{2}\right) \sin \alpha \cos \alpha \\
\left(\varepsilon_{1}-\varepsilon_{2}\right) \sin \alpha \cos \alpha & \varepsilon_{1} \sin ^{2} \alpha+\varepsilon_{2} \cos ^{2} \alpha
\end{array}\right],
\end{aligned}
$$

where $\varepsilon_{i}>0$ are stiffnesses along the axis $\xi^{i}$ inclined at angle $\alpha$.

\section{Remark:}

The trajectory of a block is a straight line inclined by the angle $\varphi$. Moreover, the observed inclination angle $\varphi$ does not depend on particular values of $\lambda_{1}, \lambda_{2}$, but only on its ratio $\lambda_{1} / \lambda_{2}$.

\subsubsection{Mechanical interpretation of the model.}

As is known, the mechanical interpretation of the regularized friction model assuming elastic deformations is a spring-slider system, see Simo and Hughes [21]. As generalization of this; according to our model, we consider a material point with two spring-slider systems, see Fig. 6 . The properties of these systems are the following: $\varepsilon_{i}$-stiffness of $i^{\text {th }}$ spring, $\mu_{i}$ - coefficient of friction for $i^{t h}$ sliding device. Each system $i$ is constrained to move parallel along the axis $X_{i}$ respectively. The constant force $\mathbf{F}$ inclined with angle $\alpha$ to the coordinate axis $X_{1}$ is applied to the point. Then the trajectory of the point lies either above the force line or below the force line depending on the ratio of eigenvalues $\lambda_{1}$ and $\lambda_{2}$ as discussed later, see also computational analysis in [15].

\section{Calibration of parameters for different models}

As a representative parameter we take the curve $\varphi(\alpha)$ known from the experiment, see Fig. 4. In addition, we distinguish two orthotropy angles: $\alpha-$ orthotropy angle for the adhesion tensor $\mathbf{B}$ and $\beta$ - orthotropy angle for the friction tensor $\mathbf{F}$. In order to unify the computations we chose the orthotropic values as $\mu_{1}<\mu_{2}$ and $\varepsilon_{1}<\varepsilon_{2}$. The following test computations are performed for calibration purposes:

1. The orthotropic friction model as discussed in Section 3.1. 


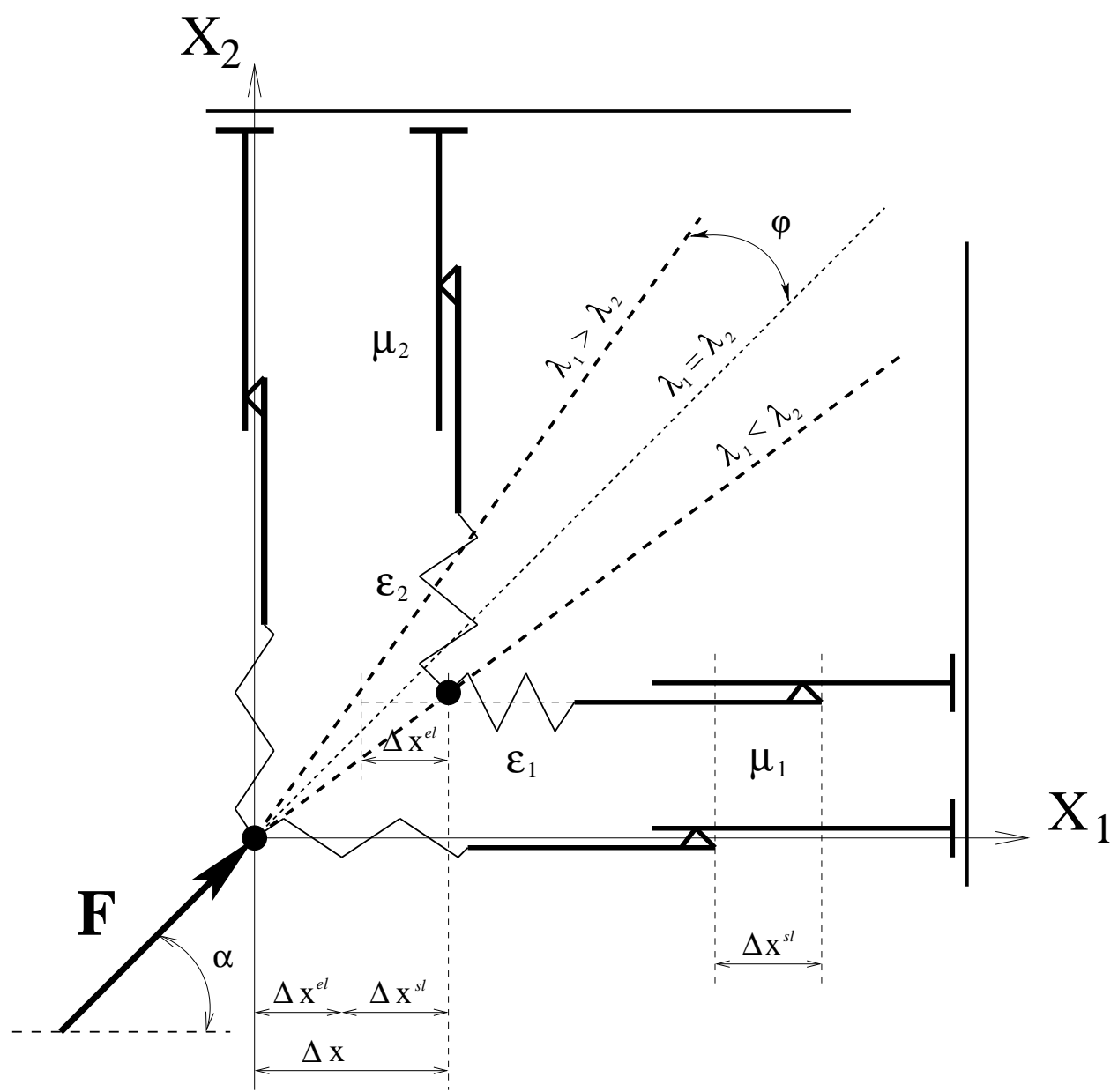

Figure 6: Mechanical interpretation of the orthotropic adhesion - orthotropic friction model. A material point on a plane with a two spring - two slider system loaded by the force $\mathbf{F}$ in plane.

2. The interface model including coupled orthotropy for both adhesion and friction as discussed in Section 3.2 with the specific case of isotropic adhesion $\mathbf{B}=-\varepsilon \mathbf{E}$.

3. The interface model including coupled orthotropy for both adhesion and friction with the specific case of isotropic friction $\mathbf{F}=\frac{1}{\mu^{2}} \mathbf{E}$.

4. The interface model including orthotropy for both adhesion and friction with the specific case of coinciding orthotropy angle $\alpha=\beta$.

5. The interface model including orthotropy for both adhesion and friction with the specification of the friction orthotropy angle $\beta$ by $90^{\circ}$ degrees as $\beta=\alpha+\pi / 2$. 


\subsection{Case 1}

We start the validation from the simple model including only orthotropic friction as discussed in Sect. 3.1. Our aim is to find out a case describing qualitatively the experimental results. Therefore, we perform a test computation with the following friction coefficients $\mu_{1}=0.1, \mu_{1}=0.5$. In Fig. 7 the results are depicted. In addition, at an extremal angle $\alpha=\arctan \frac{\mu_{2}}{\mu_{1}}=\left(78.69^{\circ}\right)$ the maximum value $\varphi_{\max }=\arctan \frac{\mu_{2}^{2}-\mu_{1}^{2}}{2 \mu_{1} \mu_{2}}=\left(67.38^{\circ}\right)$ is computed by analyzing the shape of a curve. It can be seen, that the point is moving into the direction with a smaller friction coefficient, which contradicts the experimental curve, see Fig. 4.

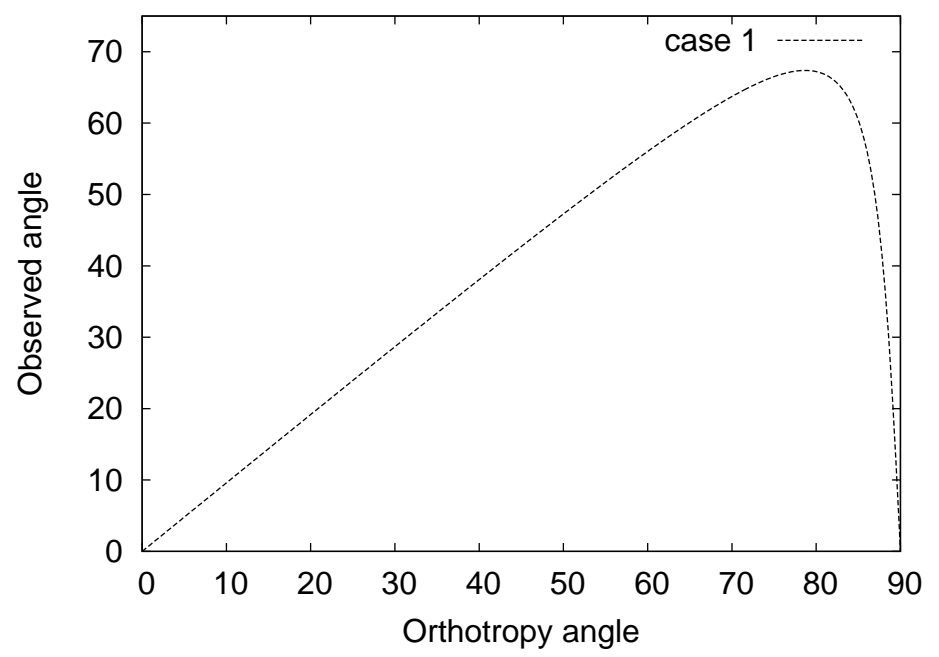

Figure 7: Computed inclination angle vs. orthotropy angle. Case 1: Purely orthotropic friction model.

\subsection{Case 2}

As a next step, we choose the orthotropic adhesion - orthotropic friction interface model, but including orthotropy only for the friction tensor and keeping the adhesion tensor to be isotropic, $\mathbf{B}=-\varepsilon_{T} \mathbf{E}$. In this case, a structure of the matrix in eqns. (21) and (24) is given as $\mathbf{A}=-\varepsilon_{T}^{2} \mathbf{F}$ leading to the inclination angle in eqn. (25) to be defined as

$$
\tan \varphi=\frac{\left(\mu_{1}^{2}-\mu_{2}^{2}\right) \tan \beta}{\mu_{1}^{2}+\mu_{2}^{2} \tan ^{2} \beta} .
$$

The analysis with the values $\mu_{1}=0.1$ and $\mu_{2}=0.5$ gives the curve presented in Fig. 8 with the extremal parameters $\beta_{e x t}=11.31^{\circ}$ and $\varphi_{\min }=-67.38^{\circ}$. In 
this case, the point tends to move into the direction with the larger friction coefficient. This is also a contradiction to the experimental results.

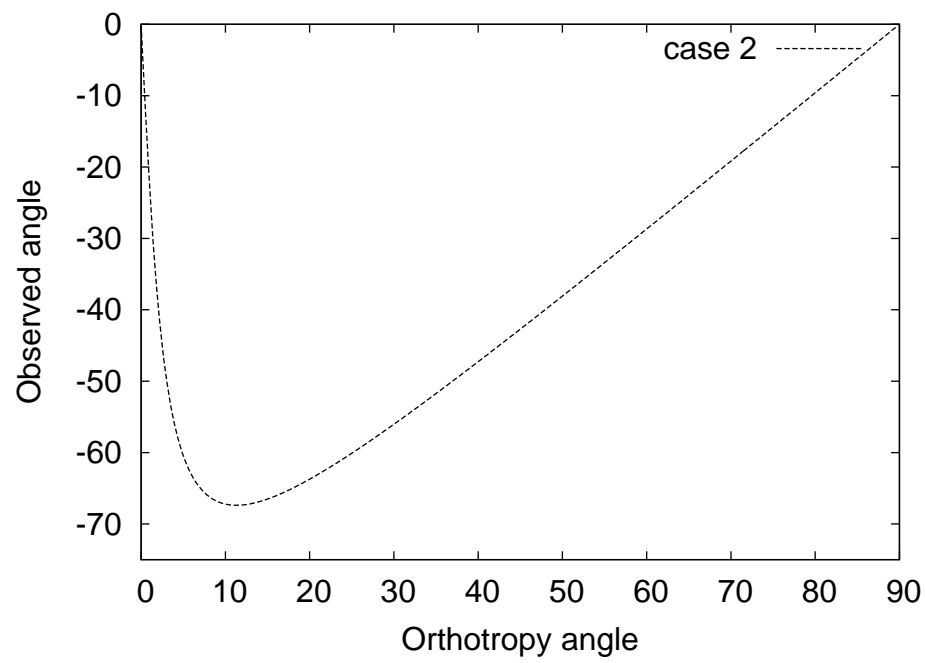

Figure 8: Orthotropic adhesion - orthotropic friction interface model. Computed inclination angle vs. orthotropy angle. Case 2: isotropic adhesion - orthotropic friction.

\subsection{Case 3}

As a next step, within the orthotropic adhesion - orthotropic friction interface model orthotropy is assumed only for adhesion with parameters $\varepsilon_{1}=1 \cdot 10^{4}$ and $\varepsilon_{2}=5 \cdot 10^{4}$. The following structure of the matrix $\mathbf{A}$ is obtained

$$
\mathbf{A}=\mathbf{B F B}=\frac{1}{\mu^{2}} \mathbf{B}
$$

leading to an inclination angle $\varphi$ defined as

$$
\tan \varphi=\frac{\left(\varepsilon_{2}^{2}-\varepsilon_{1}^{2}\right) \tan \alpha}{\varepsilon_{2}^{2}+\varepsilon_{1}^{2} \tan ^{2} \alpha}
$$

The result is depicted in Fig. 9 with the extremal values as $\alpha_{e x t}=78.69^{\circ}$ and $\varphi_{\max }=67.38^{\circ}$. The curve shows a motion into the direction with the smaller stiffness $\varepsilon_{1}$. This contradicts the experimental results as well.

\section{Summary}

We observed that neither the purely orthotropic friction model, nor the coupled model with separately included orthotropy either for friction, or for adhesion is unable to capture the phenomena even qualitatively.

\subsection{Case 4}

Now we keep the orthotropy for both the adhesion tensor and the friction tensor with the same angle, namely $\alpha=\beta$. The structure of the tensor $\mathbf{A}$ is found 


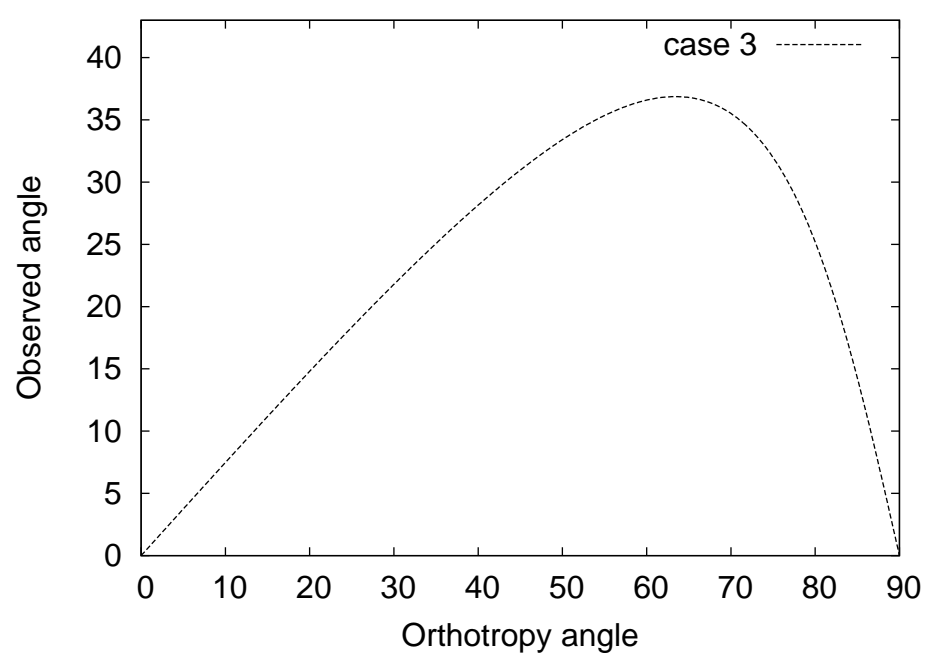

Figure 9: Orthotropic adhesion - orthotropic friction interface model. Computed inclination angle vs. orthotropy angle. Case 3: orthotropic adhesion - isotropic friction.

from the spectral decomposition in eqns. (6) and (30) with $\mathbf{Q}_{\alpha} \equiv \mathbf{Q}_{\beta}$

$$
\mathbf{A}=\mathbf{B F B}=\mathbf{Q}_{\alpha} \boldsymbol{\Lambda}_{B} \underbrace{\mathbf{Q}_{\alpha}^{T} \mathbf{Q}_{\beta}}_{\mathbf{E}} \boldsymbol{\Lambda}_{F} \underbrace{\mathbf{Q}_{\beta}^{T} \mathbf{Q}_{\alpha}}_{\mathbf{E}} \boldsymbol{\Lambda}_{B} \mathbf{Q}_{\alpha}^{T}=\mathbf{Q}_{\alpha} \boldsymbol{\Lambda}_{B}^{2} \boldsymbol{\Lambda}_{F} \mathbf{Q}_{\alpha}^{T},
$$

leading to the eigenvalues $\lambda_{i}=\varepsilon_{i} / \mu_{i}$. This case gives a more comprehensive information for the analysis of the physical experiments. The most important issue is that by the combination of two orthotropic tensors an isotropic case can be recovered. This case appears if we take the value in proportion $\frac{\varepsilon_{1}}{\mu_{1}}=\frac{\varepsilon_{2}}{\mu_{2}}$ leading to $\lambda_{1}=\lambda_{2}$, e.g. with the combination of two previous cases with $\varepsilon_{1}=10^{4}$ and $\varepsilon_{2}=5 \cdot 10^{4}$ and $\mu_{1}=0.1, \mu_{2}=0.5$ isotropy of the motion is recovered. For $\lambda_{1}<\lambda_{2}$ we obtain a behavior similar to case 3 ; for $\lambda_{1}>\lambda_{2}$ we find a behavior similar to case 2. E.g. the computation with the parameters $\varepsilon_{1}=0.5 \cdot 10^{4}, \varepsilon_{2}=5 \cdot 10^{4}$ and $\mu_{1}=0.1, \mu_{2}=0.5$ shows similarity to case 3 as shown in Fig. 10. In this computational case, we assumed that the ripples are softer in direction $X_{1}$, see Fig. 2, which is obviously not the case even without measurements of the ripple stiffnesses.

\subsection{Case 5}

Finally, we can choose the last possible modification for the observed geometrical orthotropy for surfaces as taken in the experiment. We define a new angle $\hat{\beta}$ as a main angle of surface asperities in the experiment, see Fig. 11. The orthotropy angle $\beta$ for the friction tensor is shifted by $90^{\circ}$ degrees to $\beta=\alpha+\pi / 2$ with respect to the orthotropy angle $\alpha$ for the adhesion tensor. The structure of the tensor $\mathbf{A}$ is given according to eqn. (34), but now the composition of the two orthogonal matrices $\mathbf{Q}_{\alpha}^{T} \mathbf{Q}_{\beta}$ leads to: 


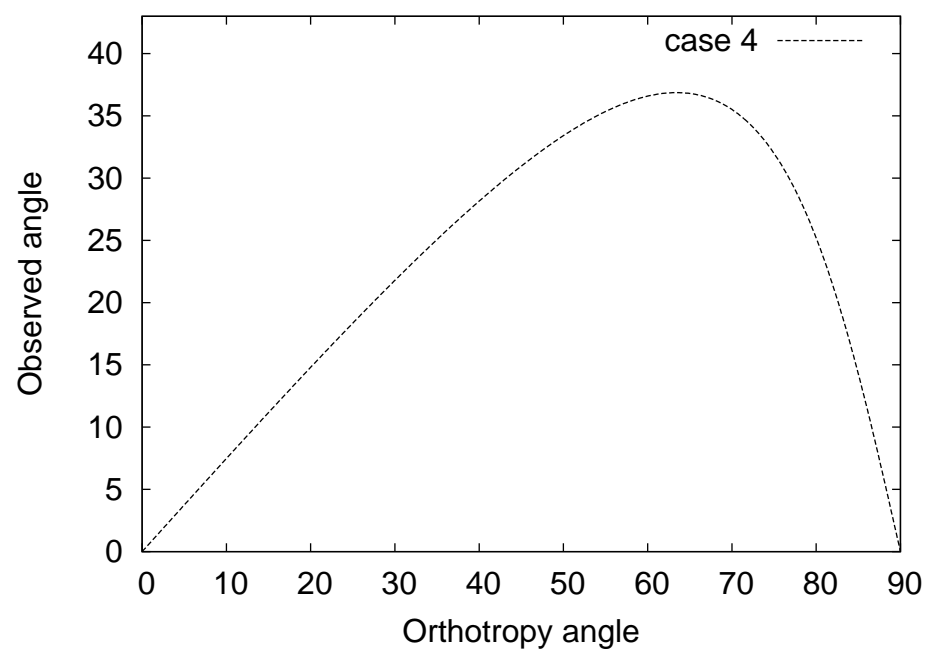

Figure 10: Orthotropic adhesion - orthotropic friction interface model. Computed inclination angle vs. orthotropy angle. Case 4: orthotropic adhesion - orthotropic friction with eigenvalues $\lambda_{1}<\lambda_{2}$.

$$
\begin{aligned}
\mathbf{Q}_{\alpha}^{T} \mathbf{Q}_{\beta} & =\left[\begin{array}{cc}
\cos \alpha & -\sin \alpha \\
\sin \alpha & \cos \alpha
\end{array}\right]^{T} \cdot\left[\begin{array}{cc}
\cos \beta & -\sin \beta \\
\sin \beta & \cos \beta
\end{array}\right]= \\
& =\left[\begin{array}{cc}
\cos \alpha & -\sin \alpha \\
\sin \alpha & \cos \alpha
\end{array}\right]^{T} \cdot\left[\begin{array}{cc}
-\sin \alpha & -\cos \alpha \\
\cos \alpha & -\sin \alpha
\end{array}\right]= \\
& =\left[\begin{array}{cc}
0 & -1 \\
1 & 0
\end{array}\right] .
\end{aligned}
$$

Then, the matrix $\mathbf{A}$ in eqn. (34) is derived as

$$
\begin{aligned}
& \mathbf{A}=\mathbf{B F B}=\mathbf{Q}_{\alpha} \boldsymbol{\Lambda}_{B} \underbrace{\mathbf{Q}_{\alpha}^{T} \mathbf{Q}_{\beta}} \Lambda_{F} \underbrace{\mathbf{Q}_{\beta}^{T} \mathbf{Q}_{\alpha}} \Lambda_{B} \mathbf{Q}_{\alpha}^{T} \\
& =\mathbf{Q}_{\alpha}\left[\begin{array}{cc}
\frac{\varepsilon_{1}^{2}}{\mu_{2}^{2}} & 0 \\
0 & \frac{\varepsilon_{2}^{2}}{\mu_{1}^{2}}
\end{array}\right] \mathbf{Q}_{\alpha}^{T},
\end{aligned}
$$

leading to the following eigenvalues $\lambda_{1}=\varepsilon_{1} / \mu_{2}$ and $\lambda_{2}=\varepsilon_{2} / \mu_{1}$ in eqn. (24). The computation with $\varepsilon_{1}=10^{4}$ and $\varepsilon_{2}=10^{3}$ and $\mu_{1}=0.1, \mu_{2}=0.5$ gives the curve $\varphi$ vs. $\hat{\beta}$ depicted in Fig. 12, which quantitively has a shape similar to the experimental one (maximum from the left side). The extremal values are found as $\hat{\beta}_{\text {ext }}=26.56^{\circ}$ and $\varphi_{\max }=36.87^{\circ}$.

Thus, summarizing the numerical investigations and focusing on the comparison to the experiments it becomes obvious that for the surface as given in 
the experiment it is necessary to apply the orthotropic adhesion - orthotropic friction interface model.

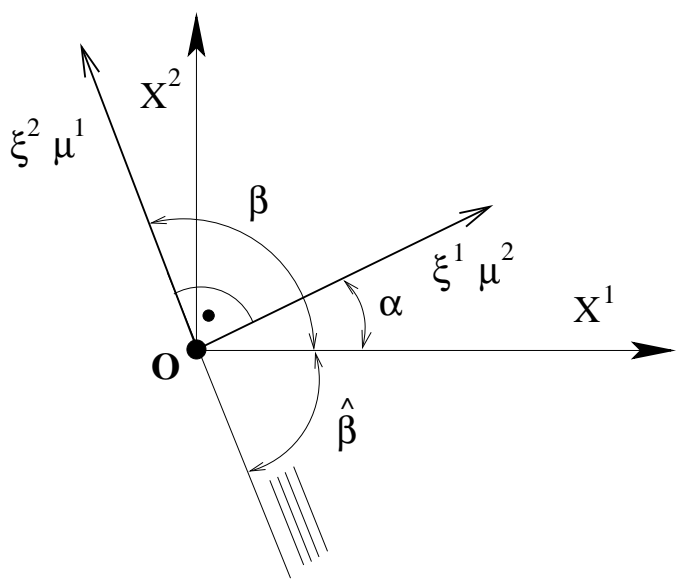

Figure 11: Definition of the experimentally observed angle $\hat{\beta}$, by an orthotropy angle $\alpha$ for the adhesion tensor and an orthotropy angle $\beta$ for the friction tensor.

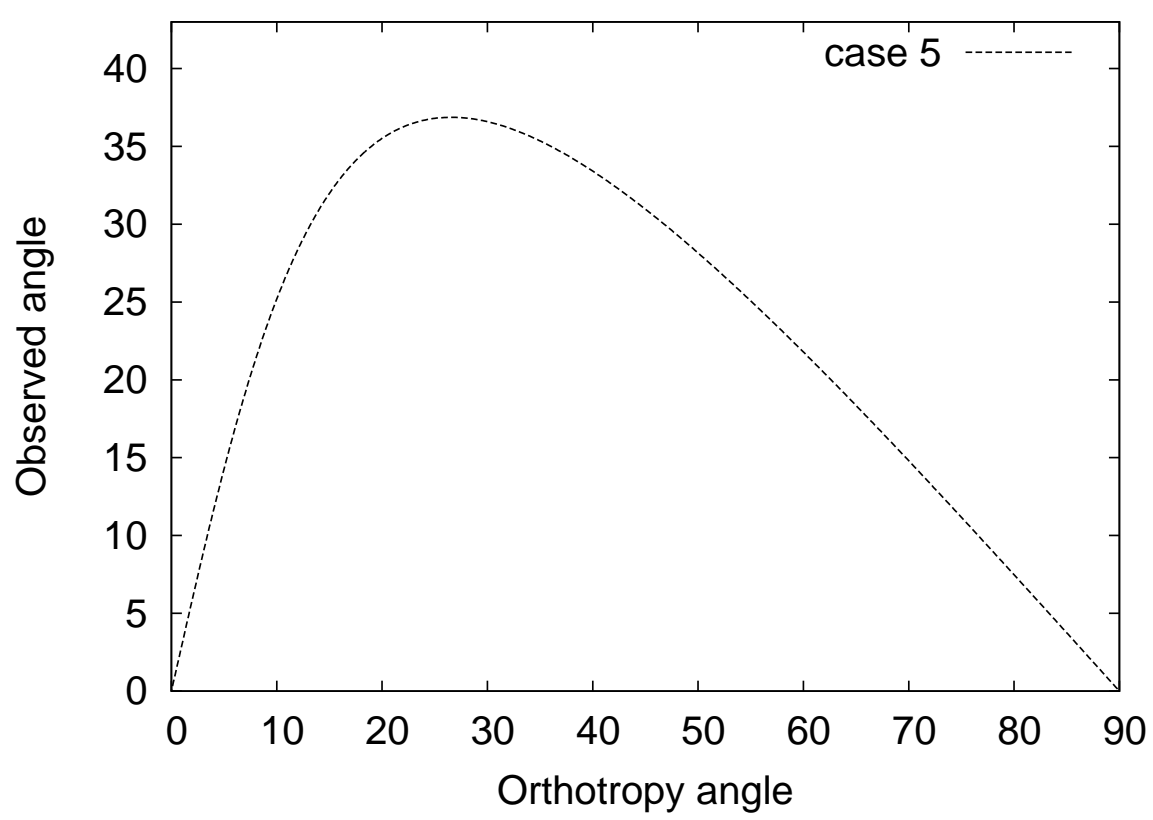

Figure 12: Orthotropic adhesion - orthotropic friction interface model. Computed inclination angle $\varphi$ vs. redefined orthotropy angle $\hat{\beta}$. Case 5: orthotropic adhesion - orthotropic friction with angles $\beta=\alpha+\frac{\pi}{2}$.

In this case, the force $T_{2}$ measured in direction $X_{2}$ is larger, while the ripples are softer in the same direction, which is visually observed in experiments even without measurement of ripple stiffnesses, see Fig. 2. Moreover, assuming $\frac{\varepsilon_{1}}{\varepsilon_{2}}=\frac{\mu_{1}}{\mu_{2}}$ we obtain $\varphi(\alpha)=0$, i.e. the block is no longer inclined, and we recover the geometrically isotropic behavior already observed in experiments, see Sect. 2.2.1. In this case, orthotropy for friction is compensated by orthotropy for adhesion leading to the observed isotropic behavior of the block, though the 
reason for this remains uncovered.

\subsection{Calibration of the theoretical curve by extremal values}

As found from the proposed model, the inclination angle $\varphi$ depends only on the ratio of eigenvalues $\lambda_{1} / \lambda_{2}$, see eqn. (25). This ratio contains information also about the ratio of adhesion parameters $\varepsilon_{1} / \varepsilon_{2}$ :

$$
\text { ratio }_{e x t}=\frac{\lambda_{1}}{\lambda_{2}}=\frac{\varepsilon_{1}}{\varepsilon_{2}} \cdot \frac{\mu_{1}}{\mu_{2}} .
$$

Since, the friction coefficients $\mu_{1}, \mu_{2}$ are defined via the measurement of forces in the experiment, the inclination angle $\varphi$ depends only on the ratio of eigenvalues $\varepsilon_{1} / \varepsilon_{2}$, see also the Remark in Section 3.2.1. This fact gives the possibility to judge macro properties for ripple stiffnesses without measurements. Thus, we will use eqn. (25) for a calibration of the model. Calibration is provided according to following rules: 1) a maximum rule - both theoretical and experimental curves must achieve the same maximum; 2) a least square fit method. The friction coefficients have been determined previously to $\mu_{1}=0.408, \mu_{2}=0.625$.

According to the maximum rule the value for the angle $\varphi_{\text {ext }}$ defined in eqn. (29) is used for calibration purposes. Taking e.g. the maximum angle $\varphi_{\max }=11.5^{\circ}$ measured for the velocity $12.5 \mathrm{~cm} / \mathrm{sec}$ the ratio of the eigenvalues given in eqn. (29) becomes

$$
\text { ratio }_{e x t}=\frac{\lambda_{1}}{\lambda_{2}}=-0.20345 \pm 1.02048=0.817,
$$

where only the positive solution is taken. The ratio of the stiffness coefficient is then obtained as

$$
\frac{\varepsilon_{1}}{\varepsilon_{2}}=\frac{\mu_{2}}{\mu_{1}} \cdot \text { ratio }_{\text {ext }}=\frac{0.625}{0.408} \cdot 0.817=1.251
$$

A more mathematically precise least square fit method leads to the statement derived from eqn. (25). The following sum must be minimized:

$$
\begin{gathered}
\sum_{k=1}^{N}\left\{\tan \varphi^{(k)}+\frac{\left(\lambda_{1}^{2}-\lambda_{2}^{2}\right) \tan \beta^{(k)}}{\lambda_{1}^{2} \tan ^{2} \beta^{(k)}+\lambda_{2}^{2}}\right\}^{2}= \\
=\sum_{k=1}^{N}\left\{\tan \varphi^{(k)}+\frac{\left(r_{\lambda}^{2}-1\right) \tan \beta^{(k)}}{r_{\lambda}^{2} \tan ^{2} \beta^{(k)}+1}\right\}^{2} \longrightarrow \text { min }
\end{gathered}
$$

where $\varphi^{(k)}$ are measured declination angles vs. applied orthotropy angles $\alpha^{(k)}$ and $\beta^{(k)}=\pi / 2-\alpha^{(k)}$. Minimization with regard to the variable $r_{\lambda}^{2}$ leads to the 
following expression:

$$
r_{\lambda}^{2}=\frac{\sum_{k=1}^{N}\left\{\tan \beta^{(k)}\left(1+\tan ^{2} \beta^{(k)}\right)\left(\tan \beta^{(k)}-\tan \varphi^{(k)}\right)\right\}}{\sum_{k=1}^{N}\left\{\tan \beta^{(k)}\left(1+\tan ^{2} \beta^{(k)}\right)\left(\tan \beta^{(k)}+\tan \varphi^{(k)}\right)\right\}} .
$$

The cases including the computed parameters are shown in Fig. 13 and do not exhibit a quantitively good correlation, though we reached the aim to show the necessity of coupling both orthotropy for friction and orthotropy for adhesion in addition to the general applicability of the proposed model. Among the possible reasons for the bad correlations might be the following:

a) a too simple linear elastic model for adhesion region;

b) the simple Coulomb friction model for the friction region without e.g. hysteresis etc.

Nevertheless, it seems to be important to consider fairly complex models including the coupled orthotropy for the adhesion and the friction in order to describe the observed phenomena more precisely.

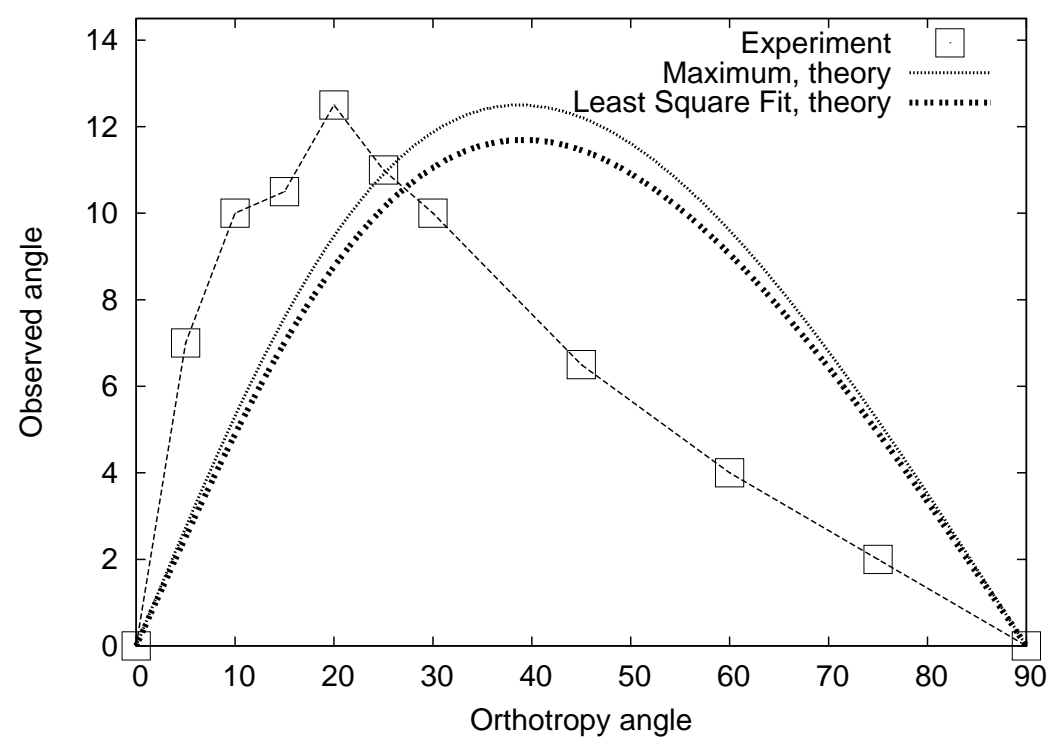

Figure 13: Observed inclination angle $\varphi$ vs. redefined orthotropy angle $\hat{\beta}$. Calibration: (1) by the absolute maximum value for different velocities of the block; (2) by the least square fit method. 


\section{Acknowledgments}

We thank mechanics master $\mathrm{W}$. Wendler for the careful preparation and performing of the experiments. His actions were very creative and are greatfully acknowledged. We also thank the DFG for the support given by grant SCHW 307/18-2.

\section{Conclusions}

The necessity to apply a coupled model including anisotropy for both adhesion and friction is shown for particular soft anisotropic surfaces, such as a periodic wavy rubber profile. The macro characteristics of the proposed model such as macro friction coefficients $\mu_{1}, \mu_{2}$ can be defined via the measurement of global forces, while the trajectory, namely, the observed sliding angle $\varphi(\alpha)$ gives information about the ratio of orthotropic stiffnesses $\varepsilon_{1} / \varepsilon_{2}$. The model does not show the dependence of particular values of stiffnesses $\varepsilon_{1}$ and $\varepsilon_{2}$ on the trajectory, which was also confirmed by experiments. The model allows to skip measurements of surface microstiffnesses. Moreover, particular phenomena when a sliding block shows geometrically isotropic behavior can be described correctly with the proposed model.

The considered model contains only the coupling of linear models for adhesion and friction, which is a possible explanation for the rather poor quantitative correlation with experiments. The key to achieve better correlation - from our point of view - can be the coupling of a more general law for adhesion as well as for friction. Thus, a more complex elastic law (e.g. Ogden material law for 2D case) can be taken for the adhesion region together with a more complex friction law for the friction region (e.g. see the proposals of He and Curnier [11] and recently Zmitrowicz [31]). The calibration process, in due course, can be provided by experimental investigations as well as by numerical tests involving homogenization processes and multi-scale techniques, for which the methodology for the isotropic case is shown in [1]. 


\section{References}

[1] Bandeira, A. A., Wriggers, P., Pimenta, P. M. Numerical derivation of contact mechanics interface laws using a finite element approach for large 3D deformation. International Journal for Numerical Methods in Engineering. 59 (2004) 173-195.

[2] Bandeira, A. A., Pimenta, P. M., Wriggers, P. A study of contact surface microstructure using a homogenization procedure considering elastoplastic behaviour of the asperities. 4th Contact Mechanics International Symposium. CMIS 2005, Hannover.

[3] Borwein, J. M., Lewis, A. S. Convex analysis and nonlinear optimization. Theory and examples (Springer: New York, Heidelberg, 2000).

[4] Buczkowski, R., Kleiber, M. Elasto-plastic interface model for 3D-frictional orthotropic contact problems. International Journal for Numerical Methods in Engineering. 40 (1997) 599-619.

[5] Buczkowski, R., Kleiber, M. A stochastic model of rough surfaces for finite element contact analysis. Computer Methods in Applied Mechanics and Engineering. 199 (1999) 43-59.

[6] Buczkowski, R., Kleiber, M. Statistical model of strongly anisotropic rough surfaces for finite element contact analysis. International Journal for $\mathrm{Nu}$ merical Methods in Engineering. 49 (2000) 1169-1189.

[7] Carbone. G., Mangialardi, L. Adhesion and friction of an elastic half-space in contact with a slightly wavy rigid surface. Journal of the Mechanics and Physics of Solids. 52 (2004) 1267-1287.

[8] Curnier, A. A theory of friction. International Journal of Solids and Structures. 20 (1984) 637-647.

[9] Greenwood, J. A., Williamson, J. B. P. Contact of nominally flat surfaces. Proc. of the Royal Society of London. Series A, Mathematical and Physical Sciences. 295 (1966) 300-319.

[10] Greenwood, J. A. A unified theory of surfaces roughness. Proc. of the Royal Society of London. Series A, Mathematical and Physical Sciences. 393 (1984) 133-157.

[11] He, Q.-C., Curnier, A. Anisotropic dry friction between two orthotropic surfaces undergoing large displacements. European Journal of Mechanics A/Solids. 12 (1993) 631-666. 
[12] Hjiaj, M., Feng, Z.-Q., de Saxce, G., Mroz, Z. Three-dimensional finite element computations for frictional contact problems with non-associated sliding rule. International Journal for Numerical Methods in Engineering. 60 (2004) 2045-2076.

[13] Konyukhov A., Schweizerhof K. Covariant description for frictional contact problems. Computational Mechanics. 35 (2005) 190-213.

[14] Konyukhov A., Schweizerhof K. Covariant description of contact interfaces considering anisotropy for adhesion and friction. Part 1. Computer Methods an Applied Mechanics and Engineering. (published online 25 May 2006).

[15] Konyukhov A., Schweizerhof K. Covariant description of contact interfaces considering anisotropy for adhesion and friction. Part 2. Computer Methods an Applied Mechanics and Engineering. (accepted).

[16] Laursen, T. A. Computational Contact and Impact Mechanics. Fundamentals of Modeling Interfacial Phenomena in Nonlinear Finite Element Analysis (Springer: New-York, Heidelberg, Paris, 2002).

[17] McCool, John I. Comparison of models for the contact of rough surfaces. Wear. 107 (1986) 37-60.

[18] Michalowski, R., Mroz, Z. Associated and non-associated sliding rules in contact friction problems. Archives of Mechanics. (Archiwum mechaniki stosowanej), Polish Academy of Sciences. 30 (1978) 259-276.

[19] Mroz, Z., Stupkiewicz, S. An anisotropic friction and wear model. International Journal of Solids and Structures. 31 (1994) 1113-1131.

[20] Longuet-Higgins M. S. The statistical analysis of a random, moving surface. Proc. of the Royal Society of London. Series A, Mathematical and Physical Sciences. 249 (1957) 321-387.

[21] Simo, J.C., Hughes, T.J.R. Elastoplasticity and Viscoplasticity: computational aspects, Springer-Verlag: Berlin, (1992).

[22] Whitehouse, D. J., Archard, J. F. The properties of random surfaces of significance in their contact. Proc. of the Royal Society of London. Series A, Mathematical and Physical Sciences. 316 (1970) 97-121.

[23] Whitehouse, D. J., Phillips, M. J. Two-dimensional discrete properties of random surfaces. Proc. of the Royal Society of London. Series A, Mathematical and Physical Sciences. 305 (1982) 441-468. 
[24] Wriggers, P. Computational Contact Mechanics, John Wiley \& Sons: Chichester (2002).

[25] Wriggers, P., Zavarise, G. Thermomechanical contact - a rigorous but simple numerical approach. Computer and Structures. 46 (1993) 47-53.

[26] Wriggers, P., Zavarise, G. On the application of Augmented Lagrangian techniques for nonlinear constitutive laws in contact interface. Communications in Applied Numerical Methods. 9 (1993) 815-824.

[27] Zavarise, G., Wriggers, P., Stein, E., Schrefler, B. A numerical model for thermomechanical contact based on microscopic interface laws. Mechanics Research Communications. 19 (1992) 173-182.

[28] Zmitrowicz, A. A theoretical model of anisotropic dry friction. Wear. 73 (1981) 9-39.

[29] Zmitrowicz, A. Mathematical description of anisotropic friction. International Journal of Solids and Structures. 25 (1989) 837-862.

[30] Zmitrowicz, A. An equation of anisotropic friction with sliding path curvature effects. International Journal of Solids and Structures. 36 (1999) $2825-2848$.

[31] Zmitrowicz, A. Models of kinematics dependent anisotropic and heterogenous friction. International Journal of Solids and Structures, accepted for publication, June 17, 2005. 


\section{Contents}

1 Introduction $\quad 1$

2 Experimental investigation $\quad 2$

2.1 Experimental setup . . . . . . . . . . . . . . . . 3

2.2 Experimental results . . . . . . . . . . . . . . 5

2.2.1 Geometrically isotropic observed behavior of a sliding block 5

3 Analysis of various models for anisotropic friction and applicability to the observed phenomenon. $\quad 6$

3.1 Orthotropic Coulomb friction law. . . . . . . . . . . . . 7

3.2 Model for orthotropic contact interfaces including both adhesion and friction. . . . . . . . . . . . . . . . 9

3.2.1 Analysis of the model by general spectral representation. 11

3.2.2 Mechanical interpretation of the model. . . . . . . . . . . 12

4 Calibration of parameters for different models $\quad 12$

4.1 Case $1 \ldots \ldots \ldots$. . . . . . . . . . . . . . . . . . 14

4.2 Case $2 \ldots \ldots \ldots \ldots \ldots$

4.3 Case $3 \ldots \ldots \ldots \ldots \ldots \ldots$

4.4 Case $4 \ldots \ldots \ldots \ldots \ldots \ldots$

4.5 Case $5 \ldots \ldots \ldots \ldots \ldots$

4.6 Calibration of the theoretical curve by extremal values . . . . . 19

5 Acknowledgments $\quad 21$

6 Conclusions 21 